\title{
Emerging higher-level artificial neural network-based intelligent systems
}

\author{
Sabah Mohammed ${ }^{1} \cdot$ Carlos Ramos $^{2} \cdot$ Wai Chi Fang ${ }^{3} \cdot$ Tia-hoon Kim $^{4}$ \\ Published online: 9 March 2021 \\ (C) The Author(s), under exclusive licence to Springer-Verlag London Ltd., part of Springer Nature 2021
}

Artificial intelligence (AI) including symbolic AI with deep learning neural networks represents incredibly exciting and powerful machine learning-based techniques used to solve and explain many real-world problems. While there are many different means to define intelligence and learning, they all essentially involve learning, understanding from human intuition or from data, and the application of the knowledge learned to achieve one or more goals. However, the new neurosymbolic AI attempts to solve much harder problems and learn with dramatically less data as well as to provide inherently understandable and controllable decisions and actions. While researchers have been making great progress in $\mathrm{AI}$ and machine learning, they still have not been able to give machines the special ingredient that makes them intuitive like how human think and reason. Today, machines are given greater capabilities to reason and recognize objects and spoken speech, but they struggle to identify higher semantic and pragmatic patterns and issues. With the emerging research like neurosymbolic AI [1], we can greatly increase the value of AI for the benefit of every application and use. Neurosymbolic AI would provide us with the thick data [2] and to answer higher level of questions.

This special issue aims to gather researches and practitioners working in higher-level artificial neural networkbased intelligent systems in order to present, discuss theories, approaches, frameworks, algorithms, challenges, emerging issues and share original research works and practical experiences, and provide the latest, most innovative contributions, and expectations on the future of

Sabah Mohammed

mohammed@lakeheadu.ca

Lakehead University, Thunder Bay, Canada

2 ISEP, Polytechnic of Porto, Porto, Portugal

3 National Chiao Tung University, Hsinchu City, Taiwan, ROC

4 Beijing Jiaotong University, Beijing, China artificial neural network-based intelligent systems. This special issue received more than 73 submissions and accepted 15 papers. The guest editors greatly appreciate the valuable work and constructive comments provided by the reviewers as well as would like to thank the editor-in-chief Prof. John MacIntyre of the Neural Computing and Applications journal for his encouragement and guideness.

The first accepted paper is by Jung Yoon Kim et al. who proposes the development of an intelligent healthcare management system that can help to manage the health of hypertensive patients. The system includes a wrist-worn ambulatory blood pressure monitoring device that can analyze the normality of measured blood pressures. The performance evaluation results of the proposed system verified the reliability of data acquisition as compared with the existing equipment as well as the efficiency of the intelligent healthcare system.

Daqing Gong et al. proposed an attention-based two pathways densely connected convolutional networks (ATPDenseNet) to identify the gender of handwriting. There are two pathways in ATP-DenseNet: The feature pyramid could extract hierarchical the page feature and the attention-based DenseNet (A-DenseNet) could extract the word feature by fusing convolutional block attention module (CBAM) and densely connected block. Finally, ATPDenseNet makes the final prediction combining the two pathways. Experimental results show the efficiency of ATP-DenseNet; the proposed method performs better than other researches. And the visualization of the feature maps can help us to know which part of the image contributes most to gender identity.

In this paper, Xiaofeng Li et al. presented the algorithm of large-area damaged image restoration based on a generative adversarial network. Before the image restoration, it extracted the multi-scale edge detailed information of the damaged areas to increase the accuracy of image restoration. In addition, this study constructed and analyzed the generative adversarial network model in detail and found 
the optimal generation parameters and discriminant parameters. It better trained the model, generated highquality false images, and thus greatly improved the restoration accuracy of large-area damaged images. Using the image in five types of datasets as the experimental sample to verify the proposed algorithm, the results show that the restoration effect is much better than that of other literature algorithms.

The fourth paper by Kelvin KL Wong et al., a new approach based on spatiotemporal neuromuscular descriptor and adaptive filtering technique (STDAFT), is proposed to optimally characterize multiple patterns of UE movements in poststroke patients toward providing inputs for intelligently driven motor training in the rehabilitation robotic systems. The proposed STD-AFT performance was systematically investigated and assessed in comparison with commonly adopted methods via high-density surface electromyogram recordings obtained from poststroke survivors who performed 21 distinct classes of pre-defined limb movements. Furthermore, the movement intent decoding was done using four different classification algorithms.

This paper by Dianjun Zhang et al. used two deep learning algorithms: the mask R-CNN algorithm and the faster R-CNN algorithm to build ship target feature extraction and recognition models based on deep convolutional neural networks. The established models were compared and analyzed to verify the feasibility of the target detection algorithms. In this study, 5748 remote sensing maps were selected as the dataset for experiments, and two algorithms were used to classify and extract warships and civilian ships. The authors Menggang Li et al. evaluated the characteristics of Internet cars from three dimensions: corporate brand attention, corporate development, and user reputation, and built corporate brand attention, corporate development, and user review. Among them, enterprise brand attention data mainly collect user reviews; enterprise development data mainly collect industry reports and enterprise news; and user review data mainly collect text data of user evaluations of cars on automobile forums. Because text evaluation data cannot be directly used to calculate the user's reviews, this paper uses NLP and machine learning to analyze the sentiment of user reviews.

The seventh paper by $\mathrm{Ji} \mathrm{Wu}$ et al. first notices that existing methods for the Cobb angle estimation on X-rays use the segmentation and landmark information separately. To use the combined information, they propose a multitask network that takes segmentation as an auxiliary task to estimate landmarks. It achieves higher performance than the existing methods on landmark estimation. In addition, to avoid a big angle error caused by a small landmark error, they propose a Cobb angle estimation network that uses spinal curvature described by 68 landmarks to estimate the Cobb angle instead of pivot landmarks to calculate by rules.

To solve the privacy leakage problem of deep learning in robot systems and fill the gap in robotics deep learning privacy research, in this paper, Zhili Zhang et al. presented a novel privacy-preserving image multi-classification deep learning (PIDL) model in robot systems. In PIDL, two schemes are proposed that adopt two groups of encrypted activation and cost functions-sigmoid plus cross-entropy function (PIDLSC) and softmax plus log-likelihood function (PIDLSL) - with secure calculation protocols, which are applied in a fog control center (FCC) with a non-colluding honest server by homomorphic encryption to improve the training efficiency, solve the encryption computation questions, and protect data and model privacy in robot systems. Security analysis and performance evaluation demonstrate that the proposed schemes realize security, correctness, and efficiency with low communication and computational costs.

Qiaoming Liu et al. proposed the IFO-SAP clustering method. The algorithm is combined with the idea of semisupervised learning and introduces prior pairwise constraints to adjust the similarity matrix and guide the convergence procedure. Furthermore, they propose an improved fruit optimization, which introduces the idea of fuzzy density to enhance the search abilities of the fruit individuals.

Aiming at the multi-source data fusion problem in the GDP forecasting process, this paper by Wenrui Li et al. proposes the use of CAE to perform feature extraction and feature fusion on the normalized sequence and train the XGBoost algorithm to predict GDP and evaluate the importance of various influencing factors. This paper uses CAE for feature extraction and feature fusion, which improves the efficiency and accuracy of the algorithm and quantifies the importance of various influencing factors to GDP. The synthetic feature XGBoost algorithm effectively integrates multi-source data information and obtains high GDP prediction accuracy.

This paper by Xiaojun Jia et al. is an analysis of investor sentiment in the stock market based on the BERT (bidirectional encoder representations from transformers) model. Firstly, they extracted the sentiment value from online information published by the stock investor, using the Bert model. Secondly, these sentiment values were weighted by attention for computing the investor sentiment indicator. Finally, the relationship between investor sentiment and stock yield was analyzed through a two-step cross-sectional regression validation model.

The twelfth paper by Leilei Kong et al. proposed a time segment language model (TSLM). TSLM gives a theoretical basis to estimate time characteristics. It can model a 
variety of time characteristics - the time distribution of term, the time distribution of query, and the time distribution of the document. And it shows how to use these time characteristics to estimate the query model, document model, and similarity between query and document more accurately.

In the next paper on this issue, the authors Wenjie Lu et al. proposed a CNN-BiLSTM-AM method to predict the stock closing price of the next day. This method is composed of convolutional neural networks (CNNs), bidirectional long short-term memory (BiLSTM), and attention mechanism (AM). CNN is used to extract the features of the input data. BiLSTM uses the extracted feature data to predict the stock closing price of the next day. AM is used to capture the influence of feature states on the stock closing price at different times in the past to improve the prediction accuracy.

Min Huang et al. proposed a new method of CT-LSTM, in which the prediction model is established by combining the chi-square test (CT) and long short-term memory (LSTM) network model. CT is used to determine the influencing factors of air quality. The hourly air quality data and meteorological data from January 1, 2017, to December 31, 2018, are used to train the LSTM network model. The data from January 1, 2019, to December 31, 2019, are used to evaluate the LSTM network model. The
AQI level of Shijiazhuang of Hebei Province of China from January 1, 2019, to December 31, 2019, is predicted with five methods (SVR, MLP, BP neural network, simple RNN, and this paper's new method).

In the final paper of this issue, the authors Luis Javier García Villalba et al. present a method for the authentication of images. The proposed method performs detection of copy-move alterations within an image, using the discrete cosine transform. The characteristics obtained from these coefficients allow us to obtain transfer vectors, which are grouped together. Through the use of a tolerance threshold, it is possible to determine if there are regions copied and pasted within the analyzed image.

\section{References}

1. Garcez, Artur d'Avila, Luis C. Lamb (2020) Neurosymbolic AI: the 3rd Wave. arXiv preprint arXiv:2012.05876.

2. Fiaidhi J, Mohammed S (2019) Thick data: a new qualitative analytics for identifying customer insights. IT Professional 21(3):4-13

Publisher's Note Springer Nature remains neutral with regard to jurisdictional claims in published maps and institutional affiliations. 